\title{
Linkage of rheumatoid arthritis with HLA
}

\author{
C M Deighton, P J Kelly, D J Walker
}

\begin{abstract}
Objective-To determine whether HLA exerts a variable influence on the predisposition of siblings of probands with clinically mild and severe rheumatoid arthritis (RA).

Method-Calculation of crude and adjusted odds ratios for concordance rates in sibships sharing two, one and no HLA haplotypes with a proband with clinically mild and severe RA, and HLA haplotype sharing in multiply affected sibships in the same clinical groups.

Results-Compared with a reference value of 1.0 in siblings sharing no HLA haplotypes with a proband with mild $R A$, siblings sharing two HLA haplotypes with a severely affected proband had a sibship concordance rate odds ratio of 9.7 $(95 \%$ confidence interval $2 \cdot 5$ to $38 \cdot 2)$. When adjusted for age, sex, and disease duration, the odds ratio was $7.6(1.8$ to $32 \cdot 4)$. No other sibships showed concordance rates which were significantly higher than the reference group. HLA haplotype sharing in multiply affected sibships in which the proband had severe RA deviated significantly from random (two, one, and no HLA haplotypes shared: $53 \cdot 3,40$, and $6 \cdot 7 \%$, respectively; expected 25,50 , and $25 \%$ ), whereas in sibships of probands with mild RA they did not $(14 \cdot 6$, $70 \cdot 8$, and $14 \cdot 6 \%$ ).

Conclusions-In the predisposition of siblings to RA, sharing HLA haplotypes with a proband is only important if the proband has severe RA. Mild RA is not genetically linked to the HLA region.
\end{abstract}

(Ann Rheum Dis 1993; 52: 638-642)

Department of Rheumatology, Royal Victoria Infirmary,

Newcastle upon Tyne, United Kingdom C M Deighton D J Walker

Department of Medical Statistics,

Medical School,

University of

Newcastle upon Tyne,

United Kingdom

P J Kelly

Correspondence to:

Dr C M Deighton,

Department of

Rheumatology,

City Hospital,

Hucknall Road,

Nottingham NG5 1 PB

United Kingdom.

Accepted for publication

27 May 1993
One of the few consistent findings in studies of the immunogenetics of rheumatoid arthritis (RA) in white subjects is the association with Dw4 and Dw14 subtypes of HLA-DR4 and HLA-DR1. ${ }^{1}$ There is increasing evidence to suggest, however, that HLA linked genes are more important in determining disease severity than predisposition to RA. This is based on several observations: (a) patients ascertained in community surveys, where presumably milder RA resides, have a prevalence of HLA-DR4 which is not dissimilar to the non-RA population $^{2}$; (b) patients with recent onset polyarthritis do not have the HLA-DR4 association, ${ }^{3}{ }^{4}$ suggesting that this is more of a marker of disease perpetuation; and (c) HLADR4 is present in approximately $70 \%$ of hospital ascertained patients with RA, compared with $30 \%$ of controls. The prevalence is even higher in patients with extra-articular manifestations, so that patients with Felty's syndrome are almost universally HLA-DR4 positive. $^{5}$

The association of HLA-DR with RA is almost certainly a consequence of disease susceptibility or expression genes being in linkage disequilibrium with the HLA-DR locus. The likelihood that HLA-DR itself is the predisposing locus is supported by the function of HLA-DR genes in antigen presentation, and the sequence similarities between predisposing HLA-DR specificities in key areas of the antigen binding groove, as encompassed in the shared epitope hypothesis. ${ }^{6}$ The evidence for linkage between HLA and RA resides entirely with a distortion of HLA haplotype sharing in dually affected sibling pairs, with overrepresentation of siblings sharing both HLA haplotypes. ${ }^{1}$ Not all studies concur with this observation, however.

One interpretation of the data which could explain these apparently discrepant findings is that the disorder which is currently referred to as RA includes a genetically heterogeneous group of diseases, in which severe and extraarticular manifestations are linked to the HLA region, whereas what is currently referred to as mild disease does not have a major genetic component within the HLA region. We have addressed this possibility by calculating concordance rates in sibships sharing two, one, and no HLA haplotypes with probands expressing mild or severe RA, and performed linkage analyses for the same groups.

\section{Methods}

The population ascertained for this study has been described in detail previously. ${ }^{8}$ Briefly, all patients with classical or definite $\mathrm{RA}^{9}$ attending rheumatology outpatient clinics in Newcastle upon Tyne with geographically accessible same sex siblings (irrespective of their RA disease status) were ascertained for study. The rationale for this was to control for the two inherited factors which have been associated consistently with RA-that is, sex and HLAwith a view to determining other genetic and environmental factors which might be important in the disease. The following information was obtained from each proband and sibling: age, sex, RA status, disease duration, age of RA onset, and extra-articular disease manifestations.

Each patient with RA was assessed for the clinical articular severity of their disease using the spread/severity index (SS index).$^{10}$ Each set of joints (distal, proximal, and metacarpophalangeal, wrists, elbows, shoulders, jaw, neck, lumbar spine, hips, knees, ankles, and 
metatarsophalangeal) was scored as $0=$ normal, $1=$ tender only, $2=$ swollen only, $3=$ tender and swollen, and $4=$ deformed or operated upon. In asymmetrical disease the worse side was the one that was scored. For sets of joints such as the distal interphalangeal joints, the score of the majority was the one that counted. The SS index score was calculated from the sum of the scores of all of the sets of joints (maximum score 52). Each subject completed a health assessment questionnaire (HAQ) ${ }^{11}$ to indicate their level of disability. Hand radiographs taken within one year either side of the day that the patient was assessed were available for 208 patients, and were graded using a modified Larsen index scoring from 0 (normal) to 50 (severe multiple joint destruction). ${ }^{12}$

Blood was taken for HLA-A, $-\mathrm{B}$ and $-\mathrm{Cw}$ typing by the microcytotoxicity assay using standard alloantisera. ${ }^{13}$ On the basis of this, HLA haplotypes were assigned, and haplotype sharing of siblings with the proband determined as described previously. ${ }^{8}$ Measurement of IgM rheumatoid factor was performed by RAHA (Fugizoki Pharmaceutical, Tokyo, Japan) using rabbit IgG passively bound to gelatin particles. Antinuclear antibodies were tested for by a standard indirect immunofluorescent technique with Wellcome FITC antihuman immunoglobulin for labelling. HLA-DR typing was performed on 105 subjects for antigens 1-10 using $B$ lymphocytes isolated by their adherence to nylon wool columns.

Of the 240 sibships, 31 contained at least one sibling with RA in addition to the proband. The median SS index score of the proband in these multicase sibships was 21 , and this score was used to divide the group of probands into two: those with an SS index score of 21 or less (roughly the lower two thirds of the probands) and those with an SS index score greater than 21 . For the sake of convenience the lower two thirds are hereafter referred to as 'mild' RA and the upper third as 'severe' RA.

The mild and severe RA groups were compared for demographic and disease variables using the Mann-Whitney U test, $\chi^{2}$ test, and Fisher's exact test where appropriate. Crude mean sibship concordance rates were calcu- lated (as described previously ${ }^{8}$ ) for sibships sharing two, one, and no HLA haplotypes with probands in the mild and severe RA categories. Odds ratios were calculated for the sibship concordance rates using logistic regression analysis, with a reference value of 1.0 assigned to sibships in which the siblings shared no HLA haplotypes with a proband with mild RA. Adjusted odds ratios were calculated after controlling for any confounding demographic variables that were identified in the comparison of the groups with mild and severe RA. These variables were age, sex, and disease duration (see later). Linkage analysis was performed for the mild and severe proband multicase sibships using the method of Green and Woodrow. ${ }^{14}$

\section{Results}

Two hundred and forty sibships were recruited, of whom 190 were women and 50 were men. The median size of the sibships was two (including the proband) with a range of two to seven. Thirty one of the sibships included at least one non-proband with RA. Table 1 gives the demographic and disease characteristics of the groups with a proband with mild and severe RA. The subjects in the group with severe RA were more likely to be women, older, to have had RA longer, and to have developed the disease at a younger age. They had a higher HAQ and radiographic score, with a higher prevalence of nodules, vasculitis, use of disease modifying drugs, rheumatoid factor, and antinuclear antibody titres.

Table 2 gives the crude mean sibship concordance rates for the groups with a proband with mild and severe RA. The figures for the group with mild RA were all similar to each other, irrespective of the number of HLA haplotypes shared with the proband. The concordance rates seen in the sibships sharing no and one HLA haplotype with a severely affected proband were similar to each other and to those seen in the three HLA haplotype sharing groups of the proband with mild disease. The result that stands out in the table is the concordance rate for sibships sharing the two HLA haplotypes with a severely affected

Table 1 Characteristics of the rheumatoid arthritis disease severity groups

\begin{tabular}{|c|c|c|c|c|}
\hline \multirow[t]{2}{*}{ Patient characteristic } & \multicolumn{2}{|l|}{ Severity group } & \multirow{2}{*}{$\begin{array}{l}\text { Odds ratio }(O R) \text { or } \\
\text { mean difference }(M D) \\
\text { where appropriate } \\
(95 \% \text { confidence interval) }\end{array}$} & \multirow[t]{2}{*}{$p$ Value $^{\star}$} \\
\hline & $\begin{array}{l}\text { 'Mild' } \\
(n=161)\end{array}$ & $\begin{array}{l}\text { 'Severe' } \\
(n=79)\end{array}$ & & \\
\hline Spread/severity score range & $4-21$ & $22-39$ & & \\
\hline $\begin{array}{l}\text { Demographic and disease details } \\
\text { Women (No (\%)) } \\
\text { Mean (SD) age (years) } \\
\text { Mean (SD) disease duration (years) } \\
\text { Mean (SD) age of disease onset (years) } \\
\text { Mean (SD) HAQ score } \\
\text { Mean (SD) modified Larsen radiographic score }\end{array}$ & $\begin{array}{l}118(73) \\
54 \cdot 9(12 \cdot 3) \\
8 \cdot 6(8 \cdot 1) \\
46 \cdot 2(13 \cdot 7) \\
1 \cdot 4(0 \cdot 8) \\
17 \cdot 5(8 \cdot 0)\end{array}$ & $\begin{array}{l}73(92) \\
60 \cdot 6(10 \cdot 2) \\
19 \cdot 2(12 \cdot 0) \\
41 \cdot 4(13 \cdot 9) \\
2 \cdot 3(0 \cdot 5) \\
32 \cdot 8(8 \cdot 7)\end{array}$ & $\begin{array}{l}\mathrm{OR}=4 \cdot 4(1 \cdot 8 \text { to } 10 \cdot 9) \\
\mathrm{MD}=5 \cdot 7(2 \cdot 4 \text { to } 9 \cdot 0) \\
\mathrm{MD}=10 \cdot 6(7 \cdot 6 \text { to } 13 \cdot 6) \\
\mathrm{MD}=4 \cdot 8(1 \cdot 0 \text { to } 8 \cdot 6) \\
\mathrm{MD}=0 \cdot 8(0 \cdot 7 \text { to } 0 \cdot 9) \\
\mathrm{MD}=15 \cdot 3(13 \cdot 0 \text { to } 17 \cdot 6)\end{array}$ & $\begin{array}{l}0.001 \\
0.001 \\
<0.0001 \\
0.01 \\
<0.0001 \\
<0.0001\end{array}$ \\
\hline $\begin{array}{l}\text { Extra-articular features and disease modifying drugs } \\
\text { Documented nodules (No }(\%)) \\
\text { Documented vasculitis (No }(\%) \text { ) } \\
\text { History of use of disease modifying drugs (No (\%)) }\end{array}$ & $\begin{array}{l}40(25) \\
2(1) \\
91(57)\end{array}$ & $\begin{array}{r}47(59) \\
8(10) \\
59(75)\end{array}$ & $\begin{array}{l}\mathrm{OR}=4 \cdot 4(2 \cdot 5 \text { to } 7 \cdot 9) \\
\mathrm{OR}=9 \cdot 0(1 \cdot 9 \text { to } 43 \cdot 2) \\
\mathrm{OR}=2 \cdot 3(1 \cdot 3 \text { to } 4 \cdot 1)\end{array}$ & $\begin{array}{l}<0.0001 \\
0.01 \\
0.01\end{array}$ \\
\hline $\begin{array}{l}\text { Autoantibody titres } \\
\text { Median rheumatoid factor titre (interquartile range) } \\
\text { Median antinuclear antibody titre (interquartile range) }\end{array}$ & $\begin{array}{l}1 / 160(0 \text { to } 1 / 320) \\
0(0 \text { to } 1 / 10)\end{array}$ & $\begin{array}{l}1 / 160(1 / 80 \text { to } 1 / 640) \\
1 / 10(0 \text { to } 1 / 40)\end{array}$ & & $\begin{array}{l}0.04 \\
0.01\end{array}$ \\
\hline
\end{tabular}

*Significance level calculated using $\chi^{2}$, Fisher's exact test, $t$ test or Mann-Whitney $U$ test where appropriate. 
Table 2 Mean sibship concordance rates (\%) for classical and definite rheumatoid arthritis for the two disease severity groups divided into sibships sharing two, one, and no HLA haplotypes with the proband

\begin{tabular}{llr}
\hline \multirow{2}{*}{$\begin{array}{l}\text { No of HLA haplotypes } \\
\text { shared with the proband }\end{array}$} & \multicolumn{2}{l}{ Disease severity group } \\
\cline { 2 - 3 } & Mild & \multicolumn{1}{l}{ Severe } \\
\hline 2 & $7 \cdot 0(3 / 43)$ & $28 \cdot 6(8 / 28)$ \\
1 & $8 \cdot 1(8 \cdot 7 / 108)$ & $9 \cdot 8(5 / 51)$ \\
0 & $4 \cdot 9(3 / 61)$ & $4 \cdot 3(1 / 23)$ \\
\hline
\end{tabular}

Values in parentheses: numerator is the total number of affected siblings weighted for the size of the sibship. Denominator is the number of available sibships. Sibships in which the proband had several siblings may appear in more than one cell within a severity group depending on the number of HLA haplotypes each sibling shared.

proband. In this category, the concordance rate was in the region of three times greater than that seen in any other group.

This observation was also seen in the calculations of the odds ratios (table 3 ), where all categories, except for those sibships sharing two HLA haplotypes with a severely affected proband, had odds ratios which were not significantly different from the reference group of those sibships sharing no HLA haplotype with a mildly affected proband. Adjusting the odds ratios for sex, age, and disease duration did not have a marked influence on the significantly higher odds ratio of the concordance rates for sibships sharing two HLA haplotypes with a severely affected proband.

Table 4 gives the distribution of HLA haplotype sharing in the groups with a proband with

Table 3 Unadjusted odds ratios (95\% confidence intervals) for sibship concordance rates in the two proband disease severity groups for sibships sharing two, one, and no HLA haplotypes, and odds ratios, adjusted for proband age, sex, and disease duration ('adjusted'), in relation to sibships sharing no HLA haplotypes with a proband with mild rehumatoid arthritis (set as $1 \cdot 0$ )

\begin{tabular}{|c|c|c|c|}
\hline \multirow{2}{*}{\multicolumn{2}{|c|}{$\begin{array}{l}\text { No of HLA haplotypes } \\
\text { shared with the proband }\end{array}$}} & \multicolumn{2}{|l|}{ Disease severity group } \\
\hline & & Mild & Severe \\
\hline \multirow[t]{3}{*}{2} & Unadjusted & $1.85(0.50$ to 6.82$) p=0.35$ & $9.70(2.46$ to 38.24$) p=0.001$ \\
\hline & $\begin{array}{l}\text { Aajusted } \\
\text { Unadjusted }\end{array}$ & $\begin{array}{l}1.93(0.33 \text { to } 7.02) p=0.33 \\
1.45(0.28 \text { to } 7.50) p=0.63\end{array}$ & $2.13(0.53$ to 8.56$) p=0.28$ \\
\hline & Adjusted & $1.42(0.28$ to 7.34$) p=0.67$ & $1.73(0.42$ to 7.11$) \mathrm{p}=0.44$ \\
\hline \multirow[t]{2}{*}{0} & Unadjusted & 1.00 & $0.92(0.09$ to 9.14$) \mathrm{p}=0.95$ \\
\hline & Adjusted & $1 \cdot 00$ & $0.70(0.07$ to 7.19$) \mathrm{p}=0.76$ \\
\hline
\end{tabular}

Table 4 Distributions of HLA haplotype sharing of dually affected sibling pairs with rheumatoid arthritis in the two disease severity groups

\begin{tabular}{lllll}
\hline $\begin{array}{l}\text { Spread/severity index } \\
\text { score of proband }\end{array}$ & $\begin{array}{l}\text { Number of } \\
\text { probands }\end{array}$ & \multicolumn{4}{l}{ No (\%) of subjects with shared haplotypes * } \\
\cline { 3 - 5 } & & 2 & 1 & 0 \\
\hline$\leqslant 21 \dagger$ & 16 & $2 \cdot 3(14)[4]$ & $11 \cdot 3(71)[8]$ & $2 \cdot 3(14)[4]$ \\
$\geqslant 22 \ddagger$ & 15 & $8.0(53)[3 \cdot 75]$ & $6 \cdot 0(40)[7 \cdot 5]$ & $1 \cdot 0(7)[3 \cdot 75]$ \\
\hline
\end{tabular}

Note: observed values are weighted for the size of the affected sibship, one sibship having three affected members, and two sibships four affected members. Each sibship therefore contributes a score of 1.0 to the observed figures.

a score of $1 \cdot 0$ to the observed figures.

Expected value in

$\ddagger \mathrm{T}=2 \cdot 1 ; \mathrm{p}=0.01$

Table 5 Distribution of HLA-DR phenotypes in the severity groups. The $p$ value was not significant in all instances ( $\chi^{2}$ or Fisher's exact test)

\begin{tabular}{|c|c|c|c|}
\hline & \multicolumn{2}{|c|}{ Severity group (No (\%)) } & \multirow{2}{*}{$\begin{array}{l}\text { Odds ratio } \\
\text { ( } 95 \% \text { confidence interval) }\end{array}$} \\
\hline & $\begin{array}{l}\text { Mild } \\
(n=65)\end{array}$ & $\begin{array}{l}\text { Severe } \\
(n=40)\end{array}$ & \\
\hline $\begin{array}{l}\text { HLA-DR4 positive } \\
\text { HLA-DR4/blank }{ }^{\star} \\
\text { HLA-DR } 1 / \mathrm{X} t\end{array}$ & $\begin{array}{r}43(66) \\
15(23) \\
9(14)\end{array}$ & $\begin{array}{l}32(80) \\
12(30) \\
3(8)\end{array}$ & $\begin{array}{l}2.1(0.6 \text { to } 5.2) \\
1.1(0.5 \text { to } 2 \cdot 8) \\
0.5(0.1 \text { to } 2.0)\end{array}$ \\
\hline
\end{tabular}

${ }^{\star}$ Highly likely to be homozygous for HLA-DR4 (see Nepom et al). ${ }^{24}$

tWhere $\mathrm{X}$ is any allele but HLA-DR4. mild and severe disease. There was no significant deviation from random expectations of 25,50 , and $25 \%$ for the sharing of two, one, and no HLA haplotypes in the group with a proband with mild RA, but a marked overrepresentation of siblings sharing both HLA haplotypes in the group with a proband with severe RA. This supported linkage between HLA and RA for the groups with a proband with severe, but not mild, RA.

Table 5 gives the distribution of principal HLA-DR alleles and genotypes for the 105 probands for whom this was available. There was no significant difference between the two severity groups of the proband.

\section{Discussion}

This study has shown that HLA linked genes are probably only important in the predisposition of siblings to RA when they are shared with a proband with severe RA. This observation appears to be independent of age, sex, and disease duration. Furthermore, there was no evidence of linkage in the group with a proband with mild RA, in contrast with the group with a proband with severe $\mathrm{RA}$.

There is no accepted definition of mild and severe RA. In deciding how to classify our probands for this study, we could have used a functional scale such as the $\mathrm{HAQ}$, or a radiographic grading score. The problems with these scales are that major interventions such as joint replacement may lead to a marked improvement in functional grade and difficulties in radiographic grading, but could lose an indication of the destructive processes that led to the intervention. Scores of radiological progression may plateau after one to three years, ${ }^{15}$ and may not record further functional decline. No single scale of disease severity is therefore ideal for measuring all aspects of what is encompassed in the concept of severe disease. We chose to use a clinical measure of the overall distribution of the disease, and the cumulative nature of joint destruction which is the experience of many hospital based patients with RA.

By ascertaining a hospital based group of consecutively attending outpatient probands with RA, there is a marked bias towards the inclusion of more severe RA when considering the broad spectrum of disease which fulfils the diagnostic criteria. The dividing line within the study group for severe and mild RA was based largely on practical aspects of the population, and the need for analysis to have a roughly even distribution of multicase sibships. From a clinical perspective, however, this dividing line is arbitrary. It succeeded in splitting the study group into a lower two thirds of 'mild' disease, and upper third of 'severe' disease. It should be emphasised, however, that the mild group were all regular outpatient attenders, and therefore themselves represent substantial morbidity, with our severe group representing the 'worst of the worst'.

The criteria for determining severe RA were successful in selecting a group of patients with RA who were distinct from the group with mild 
$\mathrm{RA}$ in many respects. It was reassuring to see that they had a significantly higher HAQ and radiographic grading score. The higher prevalence of nodules and vasculitis, and higher titres of rheumatoid factor and antinuclear antibodies are all consistent with previous studies suggesting that these variables associate with more severe disease. ${ }^{16}$ Moreover, the higher prevalence of use of disease modifying drugs in the severe group suggested that the doctors responsible for the care of these patients felt that more aggressive treatment was warranted.

The method of clinically classifying RA was also successful in producing a group with severe disease who were more likely to be women, to be older, and to have had the disease longer. Female sex has been shown in a number of studies to be associated with more disabling RA. ${ }^{17}{ }^{18}$ These variables could have accounted for differences in concordance rates between the severity groups, because women are more prone to develop $\mathrm{RA},{ }^{19}$ and older probands with longer disease duration are more likely to have older siblings who have had a longer chance to develop RA. Therefore there was a need to control for these potential confounders in interpreting the significance of differences in concordance rates. Although this adjustment reduced the odds ratios of the concordance rates of sibships sharing two HLA haplotypes with a severely affected proband, this did not exert a major influence on the significance of the difference in this study. This suggests that other factors must be operating to explain the difference, such as HLA linked genes predisposing probands to severe disease, and their HLA identical siblings to a greater propensity to developing the disease.

How can these observations be tied in with previous studies? Before the discovery of the HLA association with RA, Lawrence noted that seropositive probands with erosive disease had much higher concordance rates for disease in first degree relatives than seronegative probands with non-erosive disease. ${ }^{20}$ There is increasing evidence to support the likelihood that HLA-DR4 is more a marker of severe disease than predisposition to RA. There is also evidence that the two HLA haplotypes are important in the predisposition to RA in that the distribution of HLA-DR genotypes in groups with RA fits closely with a recessive mode of inheritance. ${ }^{21} 22$ Furthermore, compound heterozygotes of the Dw4 and Dw14 subtypes of HLA-DR4 and HLA-DR1 are more prone to develop RA than would be expected under a simple additive model, suggesting synergistic interactions between differing alleles in disease predisposition. ${ }^{23}$ HLA-DR4 Dw4/Dw14 heterozygotes may be markedly overrepresented in Felty's syndrome, suggesting that the two HLA haplotypes may also be important in disease expression. ${ }^{24}$ There is evidence that other HLA linked loci may be important in disease expression. HLADQw7 may associate with more severe and extra-articular disease, ${ }^{25} 26$ though this may be explained in part by linkage disequilibrium with HLA-DR4. ${ }^{27}$ The null allele of the C4B gene (the locus for which resides in the class III region of the major histocompatibility complex (MHC)) may be important in extraarticular disease such as Felty's syndrome. ${ }^{26}$ Other loci in the MHC, such as tumour necrosis factor, ${ }^{28}$ the 70 kilodalton heat shock protein, ${ }^{29}$ antigen processing and transport genes, ${ }^{30}$ and possibly loci exerting an influence over testosterone levels, ${ }^{31}$ may also be important in RA.

A possible interpretation of these data which is in keeping with our observations is that what is currently labelled as RA includes a range of biologically distinct disorders. At one end severe articular and extra-articular disease in the proband and familial disease in siblings is determined to a significant degree by loci within individual $\mathrm{MHCs}$, and interactions between loci on the two inherited MHCs. At the other end, mild articular and non-extraarticular disease in the proband is associated with a low level of disease in first degree relatives, primarily as a consequence of MHC linked genes playing only a minor part in disease propensity and severity. There is much evidence to suggest that the HLA-DR locus is the most important in the MHC in determining severe RA. ${ }^{1}$ Our group with severe disease had a higher prevalence of HLA-DR4 positivity, but this was not significantly different from the group with mild disease. This supports a role for HLA-DR4 in partially explaining the higher concordance rates in siblings sharing two HLA haplotypes with a severely affected proband, but also leaves plenty of room for a role for other MHC linked loci in explaining this observation. These observations also support previous work that has suggested that extra-articular RA is immunogenetically distinct from purely synovial disease. ${ }^{26}$

Although there is no accepted definition for mild and severe RA, there is broad acknowledgment that what is currently labelled as RA covers a heterogeneous spectrum of clinical disease expression. ${ }^{25}$ Research on RA often attempts to increase the homogeneity of the study group by restricting ascertainment to certain clinical groups, such as seropositive or radiologically erosive disease, or according to functional ability. By and large, this categorisation is based on disease outcomes rather than disease processes, as indeed are the current diagnostic criteria most widely used to define RA. ${ }^{32}$ As these variables are driven by disease processes, they are liable to vary with time and treatment, so that the subgroup that a patient occupies may vary more as a consequence of confounding variables rather than being a true reflection of distinct underlying pathogenetic mechanisms. What would be much more satisfactory would be if RA could be subclassified according to immutable variables which predated disease manifestations, and where there is good evidence that these variables are important in disease pathogenesis. HLA linked genes are the best candidates to date to fit the bill. This study has suggested that disease where there is evidence of HLA linkage is more likely to be clinically 
severe with extra-articular manifestations. Further work needs to be carried out to address the possibility that subclassifying RA into HLA linked and non-linked disease is practical, and a valid method of determining subgroups of RA which are sufficiently distinct to provide a biologically tenable framework for future research into this complex disorder.

This work was supported by the Arthritis and Rheumatism Council. We thank Dr A Fay of the Department of Immunology, Newcastle General Hospital, for the autoantibody results. We thank Dr J Wentzel of the Department of Human Genetics, University of Regional Blood Transfusion Service, for the HLA typing.

1 Wordsworth P The immunogenetics of rheumatoid arthritis. Curr Opin Rheumatol 1990; 3: 423-9.

2 de Jongh B M, Westedt M-L, De Vries R R P, et al. Genetic heterogeneity of rheumatoid arthritis. Dis Markers 1986 4: $29-33$.

3 Thomson W, Payton T, Ollier W E R, Symmons D P M Barrett E M, Silman A J. The frequency of DR4 and DR in newly diagnosed cases of rheumatoid arthritis and inflammatory arthritis found in a population survey [abstract]. Br $\mathcal{F}$ Rheumatol 1992; 31 (suppl 2): 84

4 Gough A, Faint J, Huissoon A, Salmon M, Emery P. The HLA DR locus has no association with early rheumatoid arthritis [abstract]. Br $\mathcal{F}$ Rheumatol 1992; 31 (suppl 2): 85.

5 Ollier W, Venables P J W, Mumford P A, et al. HLA antigen association with extra-articular RA. Tissue Antigens 1984 13: 279-91.

6 Gregersen P K, Silver J, Winchester R J. The shared epitop hypothesis. An approach to understanding the molecular genetics of susceptibility to rheumatoid arthritis. Arthritis Rheum 1987; 30: 1205-13.

7 Go R C P, Alarcon G S, Acton R T, Koopman W J, Vittor V J, Barger B O. Analyses of HLA linkage in white families with multiple cases of seropositive rheumatoid arthritis. Arthritis Rheum 1987; 30: 1115-23.

8 Deighton C M, Wentzel J, Cavanagh G, Roberts D F, Walker $\mathrm{D} J$. Contribution of inherited factors to rheumatoid arthritis. Ann Rheum Dis 1992; 51: 182-5.

9 Ropes M W, Bennett G A, Cobb S, Jacox R, Jessar R A Revision of diagnostic criteria for rheumatoid arthritis. Bull Rheum Dis 1958; 9: 175-6.

10 Walker D J, Griffiths M, Dewar P, et al. Association of MHC antigens with susceptibility to and severity of rheumatoid arthritis in multicase families. Ann Rheum Dis 1985; 44: 519-25.

11 Kirwan J R, Reeback J S. Stanford Health Assessment Questionnaire modified to assess disability in British patients with rheumatoid arthritis. Br f Rheumatol 1986; 25: 206-9.

12 Walker D J, Griffiths I D. HLA associations are with severe rheumatoid arthritis. Dis Markers 1986; 4: 121-32.

13 Dewar P J. HLA antigens. Clin Rheum Dis 1983; 9: 103-5.
14 Green J R, Woodrow J C. Sibling method for detecting HLA-linked genes in disease. Tissue Antigens 1977; 9: $31-5$

15 van der Heijde D M F M, van Leeuwen M A, van Reil P L C M, et al. Biannual radiographic assessment of hands and feet in a three year prospective follow-up of patients with early rheumatoid arthritis. Arthritis Rheum 1992; 35: 26-34.

16 van der Heijde D M F M, van Reil P L C M, van Rijswijk M H, van de Putte L B A. Influence of prognostic features on the final outcome in RA: a review of the literature. Semin Arthritis Rheum 1988; 17: 284-92.

17 Sherrer Y S, Bloch D A, Mitchell D M, Roth S H, Wolfe F, Fries J F. Disability in rheumatoid arthritis: comparison of prognostic factors across three populations. 7 Rheumatol 1987; 14: 705-9.

18 Deighton C M, Surtees D, Walker D J. Influence of the severity of rheumatoid arthritis on sex differences in health assessment scores. Ann Rheum Dis 1992; 51: 473-5.

19 Hazes J M W, Silman A R. Review of UK data on the rheumatic diseases-2. Rheumatoid arthritis. $\mathrm{Br} \mathscr{f}$ Rheumatol 1990; 29: 310-2.

20 Lawrence J S. Rheumatoid arthritis-nature or nurture? Ann Rheum Dis 1970; 29: 357-79.

21 Rigby A S, Silman A J, Voelm L, et al. Investigating the HLA component in rheumatoid arthritis: an additive (dominant) mode of inheritance is rejected, a recessive mode is preferred. Genet Epidemiol 1991; 8: 153-7

22 Deighton C M, Cavanagh G, Rigby A S, Lloyd H I Walker D J. Both inherited HLA-haplotypes are important in the predisposition to rheumatoid arthritis $\mathrm{Br}$ f Rheumatol. In press.

23 Wordsworth B P, Pile K D, Buckley J D, et al. HLA heterozygosity contributes to susceptibility to rheumatoid arthritis. Am ₹ Hum Genet 1992; 51: 585-91.

24 Nepom G T, Byers P, Seyfried C, et al. HLA genes associated with rheumatoid arthritis. Arthritis Rheum 1989; 32: 15-21.

25 Buchanan W W, Singal D P. Is there a need to reclassify adult rheumatoid arthritis? Br $\mathcal{f}$ Rheumatol 1990; 29 377-81.

26 Hillarby M C, Clarkson R, Grennan D M, et al. Immunogenetic heterogeneity in rheumatoid disease as illustrated by different $\mathrm{MHC}$ associations (DO, Dw and C4) in articular and extra-articular subsets. Brf Rheumatol 1991; 30: 5-9.

27 Lanchbury J S S, Jaeger E E M, Sansom D M, et al. Strong primary selection for the Dw4 subtype of DR4 accounts for the HLA-DQw7 association with Felty's syndrome. Hum Immunol 1991; 32: 56-64.

28 Spies T, Morton C C, Nedospasor S A, Fiers N, Pious D, Strominger J L. Genes for tumour necrosis alpha and bet are linked to the major histocompatibility complex. Pro Natl Acad Sci USA 1986; 83: 8699-703.

29 Sargent C A, Dunham I, Trowsdale J, Campbell R D. Human major histocompatibility complex contains gen for the major heat shock protein HSP70. Proc Natl Acad Sci USA 1989; 86: 1968-72.

30 Robertson M. Proteosomes in the pathway. Nature 1991 353: 300-1

31 Cutulo M, Accardo S. Sex hormones, HLA and rheumatoid arthritis. Clin Exp Rheumatol 1991; 9: 641-6.

32 Arnett F C, Edworthy S M Bloch D A et al. The American Rheumatism Association 1987 revised criteria for the classification of rheumatoid arthritis. Arthritis Rheum 1988; 31: 315-24. 